\title{
Mathematical Analysis for a Discrete Predator-Prey Model with Time Delay and Holling II Functional Response
}

\author{
Dehong Ding, ${ }^{1}$ Kui Fang, ${ }^{1}$ and Yang Zhao ${ }^{2,3}$ \\ ${ }^{1}$ College of Information Science and Technology, Hunan Agricultural University, Changsha 410128, China \\ ${ }^{2}$ Department of Electronic and Information Technology, Jiangmen Polytechnic, Jiangmen 529000, China \\ ${ }^{3}$ College of Instrumentation \& Electrical Engineering, Jilin University, Changchun 130061, China
}

Correspondence should be addressed to Dehong Ding; ddingdehong@163.com

Received 31 May 2014; Revised 19 August 2014; Accepted 28 August 2014

Academic Editor: Zbigniew Leśniak

Copyright (C) 2015 Dehong Ding et al. This is an open access article distributed under the Creative Commons Attribution License, which permits unrestricted use, distribution, and reproduction in any medium, provided the original work is properly cited.

This paper is concerned with a discrete predator-prey model with Holling II functional response and delays. Applying Gaines and Mawhin's continuation theorem of coincidence degree theory and the method of Lyapunov function, we obtain some sufficient conditions for the existence global asymptotic stability of positive periodic solutions of the model.

\section{Introduction}

In recent years, numerous studies have been carried out on predator-prey interactions using Lotka-Volterra type functional response [1]. Considering the simplification of assumptions on prey searching, prey consumption, and environmental complexity, Holling suggested three different kinds of functional response to model more realistic predatorprey interactions than what is possible with the standard Lotka-Volterra type response $[1,2]$. Many predator-prey systems with Holling type II functional response have been investigated. In particular, the periodic solutions are of great interest. During the past decades, a large number of excellent results have been reported for a lot of different predatorprey models with Holling type II functional response. For example, Ko and Ryu [3] investigated the qualitative analysis of a predator-prey model with Holling type II functional response incorporating a prey refuge. Zhou and Shi [4] considered the existence, bifurcation, and stability of positive stationary solutions of a diffusive Leslie-Gower predator-prey model with Holling type II functional responses. Liu and Yan [5] dealt with the positive periodic solutions for a neutral delay ratio-dependent predator-prey model with a Holling type II functional response. For more related work, one can see [6-25]. Dunkel [26] pointed out that feedback control item in predator-prey models depends on the population number for certain time past and also depends on the average of the population number for a period of time past. Motivated by the viewpoint, we proposed the following predator-prey model with Holling II functional response and distributed delays:

$$
\begin{aligned}
\frac{d x_{1}}{d t}= & x_{1}(t)\left[r_{1}(t)-\int_{-\infty}^{t} k_{1}(s-t) x_{1}(s) d s\right] \\
& -x_{1}(t) \int_{-\infty}^{t} \frac{k_{2}(s-t) x_{2}(s)}{1+m x_{2}(t)} d s, \\
\frac{d x_{2}}{d t}= & x_{2}(t)\left[-r_{2}(t)-\int_{-\infty}^{t} k_{3}(s-t) x_{2}(s) d s\right] \\
& +x_{2}(t) \int_{-\infty}^{t} \frac{k_{4}(s-t) x_{1}(s)}{1+m x_{1}(t)} d s,
\end{aligned}
$$

where $x_{i}(t)(i=1,2)$ stands for the prey and predator density at time $t$. For the biological meaning of model (1), one can see [27].

As pointed out in [28-35], discrete time models are more appropriate to describe the dynamics relationship among populations than continuous ones when the populations have nonoverlapping generations. What is more, we can also get more accurate numerical simulation results from the discrete-time systems. Thus it is reasonable and interesting 
to investigate discrete-time systems governed by difference equations. Following $[33,36]$, we obtain the discrete form of system (1) as follows:

$$
\begin{aligned}
x_{1}(k+1)= & x_{1}(k) \\
& \times \exp \left\{\left[r_{1}(k)-\sum_{l=0}^{+\infty} k_{1}(-l) x_{1}(k-l)\right.\right. \\
& \left.\left.\quad-\sum_{l=0}^{+\infty} \frac{k_{2}(-l) x_{2}(k-l)}{1+m x_{2}(k-l)}\right]\right\}, \\
x_{2}(k+1)= & x_{2}(k) \quad \\
& \times \exp \left\{\left[\begin{array}{r}
-r_{2}(k)-\sum_{l=0}^{+\infty} k_{3}(-l) x_{2}(k-l) \\
\left.\left.\quad+\sum_{l=0}^{+\infty} \frac{k_{4}(-l) x_{1}(k-l)}{1+m x_{1}(k)}\right]\right\},
\end{array}\right.\right.
\end{aligned}
$$

which is a discrete time analogue of system (1), where $k=$ $0,1,2, \ldots, x_{i}(k)(i=1,2)$ stands for the prey and predator density at time $k, r_{i}(k), k_{j}(k)(i=1,2, j=1,2,3,4)$ are strictly positive sequences, and $m$ is a positive constant.

In order to obtain our main results, we assume that

(H1) $r_{i}: Z \rightarrow R^{+}$is positive $\omega$-periodic; that is, $r_{i}(k+$ $\omega)=r_{i}(k)(i=1,2)$ for any $k \in Z$, where $\omega$, a fixed positive integer, denotes the common period of the parameters in system (2);

(H2) the following inequalities are satisfied:

$$
0<\sum_{l=0}^{+\infty} k_{i}(-l)<+\infty \quad(i=1,2,3,4) .
$$

The principle aim of this paper is to discuss the effect of the periodicity of the ecological and environmental parameters on the dynamics of discrete time predator-prey model with Holling II functional response and distributed delays.

The paper is organized as follows. In Section 2, applying the coincidence degree and the related continuation theorem, a series of sufficient conditions to ensure the existence of positive solutions of difference equations are given. In Section 3, by means of the method of Lyapunov function, a set of sufficient conditions for the global asymptotic stability of the model are established. Some numerical simulations are given to illustrate the theoretical results in Section 4.

\section{Existence of Positive Periodic Solutions}

Throughout the paper, we always use the notations below:

$$
I_{\omega}:=\{0,1,2, \ldots, \omega-1\}, \quad \bar{f}:=\frac{1}{\omega} \sum_{k=0}^{\omega-1} f(k),
$$

where $f(k)$ is an $\omega$-periodic sequence of real numbers defined for $k \in Z$. In order to explore the existence of positive periodic solutions of (2) and for the reader's convenience, we will first summarize below a few concepts and results without proof, borrowing from [37].

Let $X, Y$ be normed vector spaces, let $L: \operatorname{Dom} L \subset X \rightarrow$ $Y$ be a linear mapping, and let $N: X \rightarrow Y$ be a continuous mapping. The mapping $L$ will be called a Fredholm mapping of index zero if $\operatorname{dim} \operatorname{Ker} L=\operatorname{codim} \operatorname{Im} L<+\infty$ and $\operatorname{Im} L$ is closed in $Y$. If $L$ is a Fredholm mapping of index zero and there exist continuous projectors $P: X \rightarrow X$ and $Q: Y \rightarrow$ $Y$ such that $\operatorname{Im} P=\operatorname{Ker} L, \operatorname{Im} L=\operatorname{Ker} Q=\operatorname{Im}(I-Q)$, it follows that $L \mid \operatorname{Dom} L \cap \operatorname{Ker} P:(I-P) X \rightarrow \operatorname{Im} L$ is invertible. We denote the inverse of that map by $K_{P}$. If $\Omega$ is an open bounded subset of $X$, the mapping $N$ will be called $L$-compact on $\bar{\Omega}$ if $Q N(\bar{\Omega})$ is bounded and $K_{P}(I-Q) N: \bar{\Omega} \rightarrow$ $X$ is compact. Since $\operatorname{Im} Q$ is isomorphic to $\operatorname{Ker} L$, there exists an isomorphism $J: \operatorname{Im} Q \rightarrow \operatorname{Ker} L$.

Lemma 1 ([37] continuation theorem). Let L be a Fredholm mapping of index zero and let $N$ be L-compact on $\bar{\Omega}$. Suppose

(a) for each $\lambda \in(0,1)$ every solution $x$ of $L x=\lambda N x$ is such that $x \notin \partial \Omega$;

(b) $Q N x \neq 0$ for each $x \in \operatorname{Ker} L \bigcap \partial \Omega$, and $\operatorname{deg}\{J Q N, \Omega \bigcap \operatorname{Ker} L, 0\} \neq 0$.

Then the equation $L x=N x$ has at least one solution lying in Dom $L \bigcap \bar{\Omega}$.

Lemma 2 (see [33]). Let $g: Z \rightarrow R$ be $\omega$-periodic; that is, $g(k+\omega)=g(k)$; then for any fixed $k_{1}, k_{2} \in I_{\omega}$ and any $k \in Z$, one has

$$
\begin{aligned}
& g(k) \leq g\left(k_{1}\right)+\sum_{s=0}^{\omega-1}|g(s+1)-g(s)|, \\
& g(k) \geq g\left(k_{2}\right)-\sum_{s=0}^{\omega-1}|g(s+1)-g(s)| .
\end{aligned}
$$

Lemma 3. $\left(\widehat{x}_{1}(k), \widehat{x}_{2}(k)\right)$ is an $\omega$-periodic solution of $(2)$ with strictly positive components if and only if $\left(\ln \left(\widehat{x}_{1}(k)\right), \ln \left(\widehat{x}_{2}(k)\right)\right)$ is an w-periodic solution of

$$
\begin{aligned}
x_{1}(k+1)-x_{1}(k)= & r_{1}(k)-\sum_{l=0}^{+\infty} k_{1}(-l) \exp \left(x_{1}(k-l)\right) \\
& -\sum_{l=0}^{+\infty} \frac{k_{2}(-l) x_{2}(k-l)}{1+m x_{2}(k-l)}, \\
x_{2}(k+1)-x_{2}(k)= & -r_{2}(k)-\sum_{l=0}^{+\infty} k_{3}(-l) \exp \left(x_{2}(k-l)\right) \\
& +\sum_{l=0}^{+\infty} \frac{k_{4}(-l) \exp \left(x_{1}(k-l)\right)}{1+m \exp \left(x_{1}(k)\right)} .
\end{aligned}
$$

The proofs of Lemma 3 are trivial, so we omitted the details here. 
Define

$$
l_{2}=\left\{z=\left\{z(k): z(k) \in R^{2}, k \in Z\right\}\right\} .
$$

For $a=\left(a_{1}, a_{2}\right)^{T} \in R^{2}$, define $|a|=\max \left\{\left|a_{1}\right|,\left|a_{2}\right|\right\}$. Let $l^{\omega} \subset l_{2}$ denote the subspace of all $\omega$-periodic sequences equipped with the usual supremum norm $\|\cdot\|$, that is, $\|z\|=$ $\max _{k \in I_{\omega}}|z(k)|$, for any $z=\{z(k): k \in Z\} \in l^{\omega}$. It is easy to show that $l_{\omega}$ is a finite-dimensional Banach space.

Let

$$
\begin{aligned}
& l_{0}^{\omega}=\left\{z=\{z(k)\} \in l^{\omega}: \sum_{k=0}^{\omega-1} z(k)=0\right\}, \\
& l_{c}^{\omega}=\left\{z=\{z(k)\} \in l^{\omega}: z(k)=h \in R^{2}, k \in Z\right\},
\end{aligned}
$$

and then it follows that $l_{0}^{\omega}$ and $l_{c}^{\omega}$ are both closed linear subspaces of $l^{\omega}$ and

$$
l^{\omega}=l_{0}^{\omega}+l_{c}^{\omega}, \quad \operatorname{dim} l_{c}^{\omega}=2 .
$$

Next, we will be ready to establish our result.

Theorem 4. Suppose that (H1), (H2), and (H3) $\bar{r}_{1}>$ $(1 / m) \sum_{l=0}^{+\infty} k_{2}(-l)$ hold. Then system (2) has at least an $\omega$ periodic solution with positive components.

Proof. Let $X=Y=l^{\omega}$,

$$
\begin{aligned}
& (L z)(k)=z(k+1)-z(k)=\left[\begin{array}{l}
x_{1}(k+1)-x_{1}(k) \\
x_{2}(k+1)-x_{2}(k)
\end{array}\right], \\
& (N z)(k)=\left[\begin{array}{l}
f_{1}(k) \\
f_{2}(k)
\end{array}\right],
\end{aligned}
$$

where $z \in X, k \in Z$ and

$$
\begin{aligned}
f_{1}(k)= & r_{1}(k)-\sum_{l=0}^{+\infty} k_{1}(-l) \exp \left(x_{1}(k-l)\right) \\
& -\sum_{l=0}^{+\infty} \frac{k_{2}(-l) \exp \left(x_{2}(k-l)\right)}{1+m \exp \left(x_{2}(k-l)\right)}, \\
f_{2}(k)= & -r_{2}(k)-\sum_{l=0}^{+\infty} k_{3}(-l) \exp \left(x_{2}(k-l)\right) \\
& +\sum_{l=0}^{+\infty} \frac{k_{4}(-l) \exp \left(x_{1}(k-l)\right)}{1+m \exp \left(x_{1}(k)\right)} .
\end{aligned}
$$

Then it is trivial to see that $L$ is a bounded linear operator and

$$
\operatorname{Ker} L=l_{c}^{\omega}, \quad \operatorname{Im} L=l_{0}^{\omega},
$$$$
\operatorname{dim} \operatorname{Ker} L=2=\operatorname{codim} \operatorname{Im} L \text {. }
$$

It follows that $L$ is a Fredholm mapping of index zero. Define

$$
\begin{aligned}
& P y=\frac{1}{\omega} \sum_{s=0}^{\omega-1} y(s), \quad y \in X, \\
& \mathrm{Q} z=\frac{1}{\omega} \sum_{s=0}^{\omega-1} z(s), \quad z \in Y .
\end{aligned}
$$

It is not difficult to show that $P$ and $Q$ are continuous projectors such that

$$
\operatorname{Im} P=\operatorname{Ker} L, \quad \operatorname{Im} L=\operatorname{Ker} Q=\operatorname{Im}(I-Q) .
$$

Furthermore, the generalized inverse (to $L$ ) $K_{P}: \operatorname{Im} L \rightarrow$ Ker $P \bigcap$ Dom $L$ exists and is given by

$$
K_{P}(z)=\sum_{s=0}^{\omega-1} z(s)-\frac{1}{\omega} \sum_{s=0}^{\omega-1}(\omega-s) z(s)
$$

Obviously, $Q N$ and $K_{P}(I-Q) N$ are continuous. Since $X$ is a finite-dimensional Banach space, it is not difficult to show that $\overline{K_{P}(I-Q) N(\bar{\Omega})}$ is compact for any open bounded set $\Omega \subset X$. Moreover, $Q N(\bar{\Omega})$ is bounded. Thus, $N$ is $L$-compact on $\bar{\Omega}$ with any open bounded set $\Omega \subset X$.

Now we are at the point to search for an appropriate open, bounded subset $\Omega$ for the application of the continuation theorem. Corresponding to the operator equation $L z=$ $\lambda N z, \lambda \in(0,1)$, we have

$$
\begin{array}{r}
x_{1}(k+1)-x_{1}(k) \\
=\lambda\left[r_{1}(k)-\sum_{l=0}^{+\infty} k_{1}(-l) \exp \left(x_{1}(k-l)\right)\right. \\
\left.\quad-\sum_{l=0}^{+\infty} \frac{k_{2}(-l) \exp \left(x_{2}(k-l)\right)}{1+m \exp \left(x_{2}(k-l)\right)}\right], \\
x_{2}(k+1)-x_{2}(k) \\
=\lambda\left[\begin{array}{r}
-r_{2}(k)-\sum_{l=0}^{+\infty} k_{3}(-l) \exp \left(x_{2}(k-l)\right) \\
\left.+\sum_{l=0}^{+\infty} \frac{k_{4}(-l) \exp \left(x_{1}(k-l)\right)}{1+m \exp \left(x_{1}(k)\right)}\right] .
\end{array}\right.
\end{array}
$$

Suppose that $z(k)=\left(x_{1}(k), x_{2}(k)\right)^{T} \in X$ is an arbitrary solution of system (16) for a certain $\lambda \in(0,1)$; summing both sides of (16) from 0 to $\omega-1$ with respect to $k$, respectively, we obtain

$$
\begin{gathered}
\sum_{k=0}^{\omega-1}\left[\sum_{l=0}^{+\infty} k_{1}(-l) \exp \left(x_{1}(k-l)\right)\right. \\
\left.+\sum_{l=0}^{+\infty} \frac{k_{2}(-l) \exp \left(x_{2}(k-l)\right)}{1+m \exp \left(x_{2}(k-l)\right)}\right]=\bar{r}_{1} \omega, \\
\sum_{k=0}^{\omega-1}\left[\sum_{l=0}^{+\infty} k_{3}(-l) \exp \left(x_{2}(k-l)\right)\right. \\
\left.\quad-\sum_{l=0}^{+\infty} \frac{k_{4}(-l) \exp \left(x_{1}(k-l)\right)}{1+m \exp \left(x_{1}(k)\right)}\right]=\bar{r}_{2} \omega .
\end{gathered}
$$


It follows from (16) and (17) that

$$
\begin{aligned}
& \sum_{k=0}^{\omega-1}\left|x_{1}(k+1)-x_{1}(k)\right| \leq 2 \bar{r}_{1} \omega, \\
& \sum_{k=0}^{\omega-1}\left|x_{2}(k+1)-x_{2}(k)\right| \leq 2 \bar{r}_{2} \omega .
\end{aligned}
$$

In view of the hypothesis that $z=\{z(k)\} \in X$, there exist $\xi_{i}, \eta_{i} \in I_{\omega}$ such that

$$
x_{i}\left(\xi_{i}\right)=\min _{k \in I_{\omega}}\left\{x_{i}(k)\right\}, \quad x_{i}\left(\eta_{i}\right)=\max _{k \in I_{\omega}}\left\{x_{i}(k)\right\} \quad(i=1,2) .
$$

By (17), we have

$$
\begin{gathered}
\sum_{l=0}^{+\infty} k_{1}(-l) \exp \left(x_{1}\left(\xi_{1}\right)\right) \leq \sum_{l=0}^{+\infty} k_{1}(-l) \exp \left(x_{1}(k-l)\right)<\bar{r}_{1} \omega, \\
\sum_{l=0}^{+\infty} k_{3}(-l) \exp \left(x_{2}\left(\eta_{2}\right)\right) \geq \sum_{l=0}^{+\infty} k_{3}(-l) \exp \left(x_{2}(k-l)\right)>\bar{r}_{2} \omega, \\
\sum_{l=0}^{+\infty} k_{1}(-l) \exp \left(x_{1}\left(\eta_{1}\right)\right) \omega+\frac{1}{m} \sum_{l=0}^{+\infty} k_{2}(-l) \omega \geq \bar{r}_{1} \omega, \\
\sum_{l=0}^{+\infty} k_{3}(-l) \exp \left(x_{2}\left(\xi_{2}\right)\right)-\frac{1}{m} \sum_{l=0}^{+\infty} k_{4}(-l) \leq \bar{r}_{2} \omega .
\end{gathered}
$$

Thus

$$
\begin{gathered}
x_{1}\left(\xi_{1}\right)<\ln \left[\frac{\bar{r}_{1}}{\sum_{l=0}^{+\infty} k_{1}(-l)}\right]:=\alpha_{1}, \\
x_{2}\left(\eta_{2}\right)>\ln \left[\frac{\bar{r}_{2}}{\sum_{l=0}^{+\infty} k_{3}(-l)}\right]:=\beta_{2}, \\
x_{1}\left(\eta_{1}\right)>\ln \left[\frac{\bar{r}_{1}-(1 / m) \sum_{l=0}^{+\infty} k_{2}(-l)}{\sum_{l=0}^{+\infty} k_{1}(-l)}\right]:=\alpha_{2}, \\
x_{2}\left(\xi_{2}\right)<\ln \left[\frac{\bar{r}_{2}+(1 / m) \sum_{l=0}^{+\infty} k_{4}(-l)}{\sum_{l=0}^{+\infty} k_{3}(-l)}\right]:=\beta_{1} .
\end{gathered}
$$

It follows from (18), (21), and Lemma 2 that

$$
\begin{aligned}
x_{1}(k) & \leq x_{1}\left(\xi_{1}\right)+\sum_{s=0}^{\omega-1}\left|x_{1}(s+1)-x_{1}(s)\right| \\
& \leq \alpha_{1}+2 \bar{r}_{1} \omega:=\Theta_{1}, \\
x_{1}(k) & \geq x_{1}\left(\eta_{1}\right)-\sum_{s=0}^{\omega-1}\left|x_{1}(s+1)-x_{1}(s)\right|
\end{aligned}
$$

$$
\begin{aligned}
& \geq \alpha_{2}-2 \bar{r}_{1} \omega:=\Theta_{2}, \\
x_{2}(k) & \leq x_{2}\left(\xi_{2}\right)+\sum_{s=0}^{\omega-1}\left|x_{2}(s+1)-x_{2}(s)\right| \\
& \leq \beta_{1}+2 \bar{r}_{2} \omega:=\Theta_{3}, \\
x_{2}(k) & \geq x_{2}\left(\eta_{2}\right)-\sum_{s=0}^{\omega-1}\left|x_{2}(s+1)-x_{2}(s)\right| \\
& \geq \beta_{2}-2 \bar{r}_{2} \omega:=\Theta_{4} .
\end{aligned}
$$

In view of (22), we derive

$$
\begin{aligned}
& \max _{k \in I_{\omega}}\left\{x_{1}(k)\right\} \leq \max \left\{\left|\Theta_{1}\right|,\left|\Theta_{2}\right|\right\}:=\Upsilon_{1}, \\
& \max _{k \in I_{\omega}}\left\{x_{2}(k)\right\} \leq \max \left\{\left|\Theta_{4}\right|,\left|\Theta_{5}\right|\right\}:=\Upsilon_{2} .
\end{aligned}
$$

Obviously, $\Upsilon_{i}(i=1,2)$ are independent of $\lambda \in(0,1)$. Take $M=\max \left\{\Upsilon_{1}, \Upsilon_{2}\right\}+M_{0}$, where $M_{0}$ is taken sufficiently large such that $\max \left\{\left|\ln \left(x_{1}^{*}\right)\right|,\left|\ln \left(x_{2}^{*}\right)\right|\right\}<M_{0}$, where $\left(x_{1}^{*}, x_{2}^{*}\right)^{T}$ is the unique positive solution of (6). Now we have proved that any solution $z=\{z(k)\}=\left\{\left(x_{1}(k), x_{2}(k)\right)^{T}\right\}$ of $(16)$ in $X$ satisfies $\|z\|<M, k \in Z$.

Let $\Omega:=\{z=\{z(k)\} \in X:\|z\|<M\}$; then it is easy to see that $\Omega$ is an open, bounded set in $X$ and verifies requirement (a) of Lemma 1. When $z \in \partial \Omega \cap \operatorname{Ker} L, z=\left\{\left(x_{1}, x_{2}\right)^{T}\right\}$ is a constant vector in $R^{2}$ with $\|z\|=\max \left\{\left|x_{1}\right|,\left|x_{2}\right|\right\}=M$. Then

$$
Q N z=\left[\begin{array}{l}
\chi_{1} \\
\chi_{2}
\end{array}\right] \neq 0,
$$

where

$$
\begin{aligned}
& \chi_{1}=\bar{r}_{1}-\sum_{l=0}^{+\infty} k_{1}(-l) \exp \left(x_{1}\right)-\sum_{k=0}^{\omega-1} \sum_{l=0}^{+\infty} \frac{k_{2}(-l) \exp \left(x_{2}\right)}{1+m \exp \left(x_{2}\right)}, \\
& \chi_{2}=-\bar{r}_{2}-\sum_{l=0}^{+\infty} k_{3}(-l) \exp \left(x_{2}\right)+\sum_{k=0}^{\omega-1} \sum_{l=0}^{+\infty} \frac{k_{4}(-l) \exp \left(x_{1}\right)}{1+m \exp \left(x_{1}\right)} .
\end{aligned}
$$

Now let us consider homotopic $\phi\left(x_{1}, x_{2}, \mu\right)=\mu Q N z+(1-$ $\mu) G z, \mu \in[0,1]$, where

$$
G z=\left(\begin{array}{c}
\bar{r}_{1}-\sum_{l=0}^{+\infty} k_{1}(-l) \exp \left(x_{1}\right) \\
-\bar{r}_{2}-\sum_{l=0}^{+\infty} k_{3}(-l) \exp \left(x_{2}\right)
\end{array}\right)
$$


Letting $J$ be the identity mapping and by direct calculation, we get

$$
\begin{aligned}
& \operatorname{deg}\left[J Q N\left(x_{1}, x_{2}\right)^{T} ; \Omega \bigcap \operatorname{ker} L ; 0\right] \\
& =\operatorname{deg}\left[Q N\left(x_{1}, x_{2}\right)^{T} ; \Omega \bigcap \operatorname{ker} L ; 0\right] \\
& =\operatorname{deg}\left[\phi\left(x_{1}, x_{2}, 1\right) ; \Omega \bigcap \operatorname{ker} L ; 0\right] \\
& =\operatorname{deg}\left[\phi\left(x_{1}, x_{2}, 0\right) ; \Omega \bigcap \operatorname{ker} L ; 0\right] \\
& \operatorname{sign}\left\{\operatorname{det}\left[\begin{array}{cc}
\sum_{l=0}^{+\infty} k_{1}(-l) \exp \left(x_{1}^{*}\right) & 0 \\
0 & -\sum_{l=0}^{+\infty} k_{3}(-l) \exp \left(x_{2}^{*}\right)
\end{array}\right]\right\} \\
& =\operatorname{sign}\left[-\sum_{l=0}^{+\infty} k_{1}(-l) \sum_{l=0}^{+\infty} k_{3}(-l) \exp \left(x_{2}^{*}\right) \exp \left(x_{1}^{*}+x_{2}^{*}\right)\right] \\
& =-1 \neq 0 \text {. }
\end{aligned}
$$

By now, we have proved that $\Omega$ verifies all requirements of Lemma 1; then it follows that $L z=N z$ has at least one solution in $\operatorname{Dom} L \cap \bar{\Omega}$; that is to say, (6) has at least one $\omega$-periodic solution in $\operatorname{Dom} L \cap \bar{\Omega}$, say $z^{*}=$ $\left\{z^{*}(k)\right\}=\left\{\left(x_{1}^{*}(k), x_{2}^{*}(k)\right)^{T}\right\}$. Let $\bar{x}_{1}^{*}(k)=\exp \left(x_{1}^{*}(k)\right), \bar{x}_{2}^{*}(k)=$ $\exp \left(x_{2}^{*}(k)\right)$; then by Lemma 3 we know that $\bar{z}^{*}=\left\{\bar{x}^{*}(k)\right\}=$ $\left.\left\{\bar{x}_{1}^{*}(k), \bar{x}_{2}^{*}(k)\right)^{T}\right\}$ is an $\omega$-periodic solution of system (2) with strictly positive components. The proof is complete.

\section{Global Asymptotic Stability}

Let the delays be zero; then (2) takes the form

$$
\begin{array}{r}
x_{1}(k+1)=x_{1}(k) \exp \left\{\left[r_{1}(k)-\sum_{l=0}^{+\infty} k_{1}(-l) x_{1}(k)\right.\right. \\
\left.\left.-\sum_{l=0}^{+\infty} \frac{k_{2}(-l) x_{2}(k)}{1+m x_{2}(k)}\right]\right\}, \\
x_{2}(k+1)=x_{2}(k) \exp \left\{\left[\begin{array}{r}
-r_{2}(k)-\sum_{l=0}^{+\infty} k_{3}(-l) x_{2}(k) \\
\left.\left.+\sum_{l=0}^{+\infty} \frac{k_{4}(-l) x_{1}(k)}{1+m x_{1}(k)}\right]\right\} .
\end{array}\right.\right.
\end{array}
$$

In this section, we will present sufficient conditions for the global asymptotic stability of system (28).
Theorem 5. Assume that (H1) and (H2) are satisfied and furthermore suppose that there exist positive constants $v, \mu_{1}$ and $\mu_{2}$ such that

$$
\begin{aligned}
& \mu_{1}\left[\sum_{l=0}^{+\infty} k_{1}(-l)\right]-\mu_{2}\left[\frac{1}{\left(1+m x_{1}^{*}\right)^{2}}\right]>v, \\
& \mu_{2}\left[\sum_{l=0}^{+\infty} k_{3}(-l)\right]-\mu_{1}\left[\frac{1}{\left(1+m x_{2}^{*}\right)^{2}}\right]>v .
\end{aligned}
$$

Then the positive $\omega$-periodic solution of system (28) is globally asymptotically stable.

Proof. Since the delays in system (2) have no effect on the periodic solution, then system (28) has a positive solution $\left(x_{1}^{*}(k), x_{2}^{*}(k)\right)^{T}$. Now we prove below that it is uniformly asymptotically stable. First, we make the change of variable

$$
u_{i}(k)=x_{i}(k)-x_{i}^{*}(k) \quad(i=1,2) .
$$

It follows from (28) that

$$
\begin{aligned}
& u_{1}(k+1) \\
& =x_{1}(k+1)-x_{1}^{*}(k+1) \\
& =x_{1}(k) \exp \left\{\left[r_{1}(k)-\sum_{l=0}^{+\infty} k_{1}(-l) x_{1}(k)\right.\right. \\
& \left.\left.-\sum_{l=0}^{+\infty} \frac{k_{2}(-l) x_{2}(k)}{1+m x_{2}(k)}\right]\right\} \\
& -x_{1}^{*}(k) \exp \left\{\left[r_{1}(k)-\sum_{l=0}^{+\infty} k_{1}(-l) x_{1}^{*}(k)\right.\right. \\
& \left.\left.-\sum_{l=0}^{+\infty} \frac{k_{2}(-l) x_{2}^{*}(k)}{1+m x_{2}^{*}(k)}\right]\right\} \\
& =\left\{x _ { 1 } ( k ) \operatorname { e x p } \left[\left(-\sum_{l=0}^{+\infty} k_{1}(-l)\right) u_{1}(k)\right.\right. \\
& -\left(\sum_{l=0}^{+\infty} \frac{k_{2}(-l) x_{2}(k)}{1+m x_{2}(k)}\right. \\
& \left.\left.-\sum_{l=0}^{+\infty} \frac{k_{2}(-l) x_{2}^{*}(k)}{1+m x_{2}(k)}\right)\right] \\
& \left.-x_{1}^{*}(k)\right\} \frac{x_{1}^{*}(k+1)}{x_{1}^{*}(k)} \\
& =\left\{\left[1-\sum_{l=0}^{+\infty} k_{1}(-l) x_{1}^{*}(k)\right] \frac{u_{1}(k)}{x_{1}^{*}(k)}\right.
\end{aligned}
$$

$$
\left.-\frac{1}{\left(1+m x_{2}^{*}\right)^{2}} u_{2}(k)+\rho_{1}\right\} x_{1}^{*}(k+1),
$$




$$
\begin{aligned}
& u_{2}(k+1) \\
& =x_{2}(k+1)-x_{2}^{*}(k+1) \\
& =x_{2}(k) \exp \left\{\left[-r_{2}(k)-\sum_{l=0}^{+\infty} k_{3}(-l) x_{2}(k)\right.\right. \\
& \left.\left.+\sum_{l=0}^{+\infty} \frac{k_{4}(-l) x_{1}(k)}{1+m x_{1}(k)}\right]\right\} \\
& -x_{2}^{*}(k) \exp \left\{\left[-r_{2}(k)-\sum_{l=0}^{+\infty} k_{3}(-l) x_{2}^{*}(k)\right.\right. \\
& \left.\left.+\sum_{l=0}^{+\infty} \frac{k_{4}(-l) x_{1}^{*}(k)}{1+m x_{1}(k)}\right]\right\} \\
& =\left\{x _ { 2 } ( k ) \operatorname { e x p } \left[\left(-\sum_{l=0}^{+\infty} k_{3}(-l)\right) u_{2}(k)\right.\right. \\
& \left.-\left(\sum_{l=0}^{+\infty} \frac{k_{4}(-l) x_{1}(k)}{1+m x_{1}(k)}-\sum_{l=0}^{+\infty} \frac{k_{4}(-l) x_{1}^{*}(k)}{1+m x_{1}(k)}\right)\right] \\
& \left.-x_{2}^{*}(k)\right\} \frac{u_{2}^{*}(k+1)}{x_{2}^{*}(k)} \\
& =\left\{\left[1-\sum_{l=0}^{+\infty} k_{3}(-l) x_{2}^{*}(k)\right] \frac{u_{2}(k)}{x_{2}^{*}(k)}\right. \\
& \left.-\frac{1}{\left(1+m x_{1}^{*}\right)^{2}} u_{1}(k)+\rho_{2}\right\} x_{2}^{*}(k+1),
\end{aligned}
$$

where $\left\|\rho_{i}\right\| /\|u\|(i=1,2)$ converges to zero as $\|u\| \rightarrow 0$.

Define a function $V$ by

$$
V(N(k))=\mu_{1}\left|\frac{u_{1}(k)}{x_{1}^{*}(k)}\right|+\mu_{2}\left|\frac{u_{2}(k)}{x_{2}^{*}(k)}\right|,
$$

where $\mu_{1}$ and $\mu_{2}$ are all positive constants given by (34) and (35), respectively. Calculating the difference of $V$ along the solution of system (31), we get

$$
\begin{aligned}
\Delta V= & \mu_{1}\left(\left|\frac{u_{1}(k+1)}{x_{1}^{*}(k+1)}-\frac{u_{1}(k)}{x_{1}^{*}(k)}\right|\right) \\
& +\mu_{2}\left(\left|\frac{u_{2}(k+1)}{x_{2}^{*}(k+1)}-\frac{u_{2}(k)}{x_{2}^{*}(k)}\right|\right) \\
\leq & -\mu_{1}\left[\sum_{l=0}^{+\infty} k_{1}(-l)\right]\left|u_{1}(k)\right| \\
& +\mu_{1}\left[\frac{1}{\left(1+m x_{2}^{*}\right)^{2}}\right]\left|u_{2}(k)\right|
\end{aligned}
$$

$$
\begin{aligned}
& -\mu_{2}\left[\sum_{l=0}^{+\infty} k_{3}(-l)\right]\left|u_{2}(k)\right| \\
& +\mu_{2}\left[\frac{1}{\left(1+m x_{1}^{*}\right)^{2}}\right]\left|u_{1}(k)\right| \\
& \leq-\Delta_{1}\left|u_{1}(k)\right|-\Delta_{2}\left|u_{2}(k)\right|,
\end{aligned}
$$

where

$$
\begin{aligned}
& \Delta_{1}=\mu_{1}\left[\sum_{l=0}^{+\infty} k_{1}(-l)\right]-\mu_{2}\left[\frac{1}{\left(1+m x_{1}^{*}\right)^{2}}\right], \\
& \Delta_{2}=\mu_{2}\left[\sum_{l=0}^{+\infty} k_{3}(-l)\right]-\mu_{1}\left[\frac{1}{\left(1+m x_{2}^{*}\right)^{2}}\right] .
\end{aligned}
$$

It follows from the condition (29) that there exists a positive constant $\sigma$ such that if $k$ is sufficiently large and $\|u\|<\epsilon$, then

$$
\Delta V \leq-\frac{\sigma}{2}\left\{\left|u_{1}(k)\right|+\left|u_{2}(k)\right|\right\}<-\sigma \epsilon .
$$

In view of Freedman [38], we can see that the trivial solutions of (31) are uniformly asymptotically stable and so is the solution $\left\{\left(x^{*}(k), y^{*}(k)\right)^{T}\right\}$ of (28). Thus we can conclude that the positive periodic solution of (28) is globally asymptotically stable. The proof is complete.

\section{Numerical Example}

In this section, we present some numerical results of system (2) to verify the analytical predictions obtained in the previous section. Let us consider the following discrete system:

$$
\begin{aligned}
x_{1}(k+1)=x_{1}(k) \exp \{[ & 0.5+0.3 \sin k \pi \\
& -\sum_{l=0}^{+\infty} k_{1}(-l) x_{1}(k-l) \\
x_{2}(k+1)=x_{2}(k) \exp \left\{\left[\begin{array}{l}
- \\
-
\end{array}\right.\right. & \left.\left.-\sum_{l=0}^{+\infty} \frac{k_{2}(-l) x_{2}(k-l)}{1+2 x_{2}(k-l)}\right]\right\}, \\
& -\sum_{l=0}^{+\infty} k_{3}(-l) x_{2}(k-l) \\
& \left.\left.+\sum_{l=0}^{+\infty} \frac{k_{4}(-l) x_{1}(k-l)}{1+2 x_{1}(k)}\right]\right\},
\end{aligned}
$$

where $r_{1}(k)=0.5+0.3 \sin k \pi, r_{2}(k)=0.6+0.2 \sin k \pi, k_{i}(s)=$ $e^{0.5 s}(i=1,2,3,4), m=2$, and it is easy to see that all the conditions of Theorem 4 are fulfilled. Thus system (37) has at least a positive two-periodic solution (see Figures 1 and 2). 


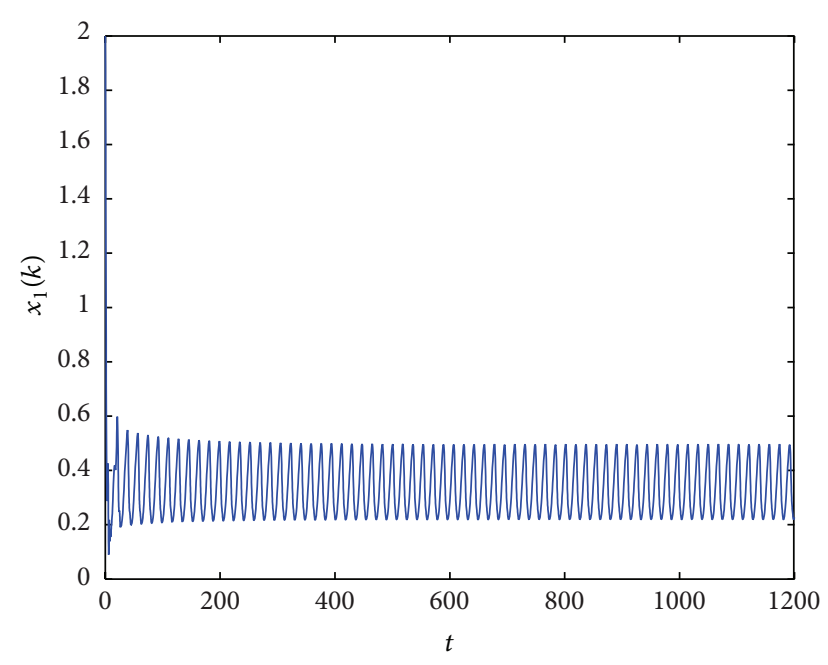

Figure 1: The time series graph of $t-x_{1}$ for system (37).

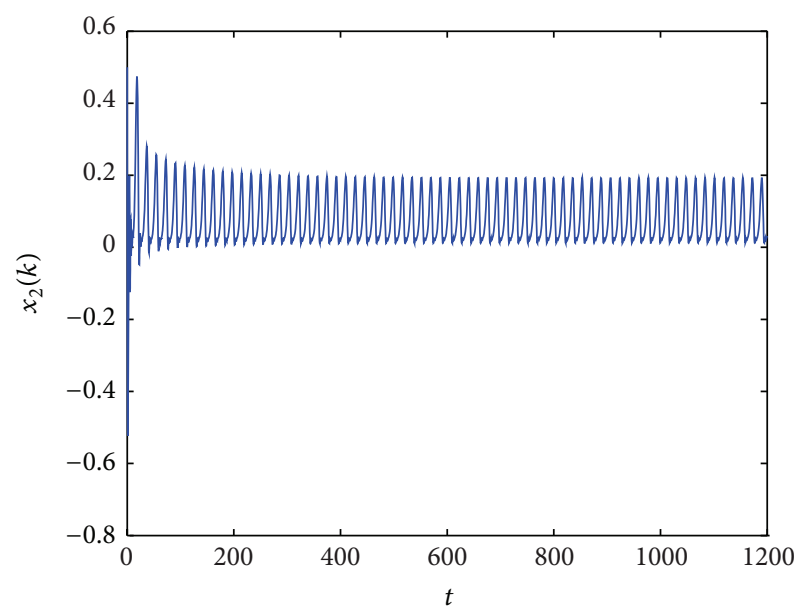

Figure 2: The time series graph of $t-x_{2}$ for system (37).

\section{Conclusions}

In this paper, a discrete predator-prey model with Holling II functional response and delays is investigated. With the aid of Gaines and Mawhin's continuation theorem of coincidence degree theory and the method of Lyapunov function, we establish some sufficient conditions for the existence and global asymptotic stability of positive periodic solutions of the model. Since the time scales can unify the continuous and discrete situations, it is meaningful to investigate the predator-prey model with Holling II functional response and delays on time scales. We leave it for future work.

\section{Conflict of Interests}

The authors declare that there is no conflict of interests regarding the publication of this paper.

\section{Acknowledgments}

This work was supported by State Science and Technology Support Program (Grant no. 2013BAD15B02), General Scientific Research Projects in Hunan Province, Department of Education, China (Grant no. 14C0542), Graduate Student Research Innovation Project of Hunan Province (Grant no. CX2014B306), and Guangxi Experiment Centre of Science and Technology (Grant no. LGZXKF201112).

\section{References}

[1] A. Priyadarshi and S. Gakkhar, "Dynamics of Leslie-Gower type generalist predator in a tri-trophic food web system," Communications in Nonlinear Science and Numerical Simulation, vol. 18, no. 11, pp. 3202-3218, 2013.

[2] P. Turchin, Complex Population Dynamics: A Theoretical/ Empirical Synthesis, vol. 35 of Monographs in Population Biology, Princeton University Press, Princeton, NJ, USA, 2003.

[3] W. Ko and K. Ryu, "Qualitative analysis of a predator-prey model with Holling type II functional response incorporating a prey refuge," Journal of Differential Equations, vol. 231, no. 2, pp. 534-550, 2006.

[4] J. Zhou and J. Shi, "The existence, bifurcation and stability of positive stationary solutions of a diffusive Leslie-Gower predator-prey model with Holling-type II functional responses," Journal of Mathematical Analysis and Applications, vol. 405, no. 2, pp. 618-630, 2013.

[5] G. R. Liu and J. R. Yan, "Positive periodic solutions for a neutral delay ratio-dependent predator-prey model with a Holling type II functional response," Nonlinear Analysis: Real World Applications, vol. 12, no. 6, pp. 3252-3260, 2011.

[6] Y. Kuang, Delay Differential Equation with Application in Population Dynamics, Academic Press, Boston, Mass, USA, 1993.

[7] H. F. Huo and W. T. Li, "Existence of positive periodic solution of a neutral impuslive delay predator-prey system," Applied Mathematics and Computation, vol. 185, no. 1, pp. 499-507, 2007.

[8] X. H. Tang, D. M. Cao, and X. F. Zou, "Global attractivity of positive periodic solution to periodic Lotka-Volterra competition systems with pure delay," Journal of Differential Equations, vol. 228, no. 2, pp. 580-610, 2006.

[9] X. Ding and F. Wang, "Positive periodic solution for a semiratio-dependent predator-prey system with diffusion and time delays," Nonlinear Analysis: Real World Applications, vol. 9, no. 2, pp. 239-249, 2008.

[10] C. V. Pao, "Global asymptotic stability of Lotka-Volterra competition systems with diffusion and time delays," Nonlinear Analysis: Real World Applications, vol. 5, no. 1, pp. 91-104, 2004.

[11] K. Gopalsamy and P. X. Weng, "Global attractivity in a competition system with feedback controls," Computers \& Mathematics with Applications, vol. 45, no. 4-5, pp. 665-676, 2003.

[12] X. S. Xiong and Z. Q. Zhang, "Periodic solutions of a discrete two-species competitive model with stage structure," Mathematical and Computer Modelling, vol. 48, no. 3-4, pp. 333-343, 2008.

[13] H. F. Huo and W. T. Li, "Periodic solution of a delayed predatorprey system without dominating instantaneous negative feedback," Applied Mathematics and Computation, vol. 156, no. 3, pp. 871-882, 2004.

[14] S. Ahmad and I. M. Stamova, "Asymptotic stability of an $N$ dimensional impulsive competitive system," Nonlinear Analysis: Real World Applications, vol. 8, no. 2, pp. 654-663, 2007. 
[15] Y. Chen and Z. Zhou, "Stable periodic solution of a discrete periodic Lotka-Volterra competition system," Journal of Mathematical Analysis and Applications, vol. 277, no. 1, pp. 358-366, 2003.

[16] M. Zhu and S. Lu, "Existence and global attractivity of positive periodic solutions of competition systems," Journal of Applied Mathematics and Computing, vol. 37, no. 1-2, pp. 635-646, 2011.

[17] M. Bohner, M. Fan, and J. Zhang, "Existence of periodic solutions in predator-prey and competition dynamic systems," Nonlinear Analysis: Real World Applications, vol. 7, no. 5, pp. 11931204, 2006.

[18] X. Liu, "Impulsive periodic oscillation for a predator-prey model with Hassell-Varley-HOLling functional response," Applied Mathematical Modelling: Simulation and Computation for Engineering and Environmental Systems, vol. 38, no. 4, pp. 1482-1494, 2014.

[19] J. Zhou and C. L. Mu, "Coexistence states of a Holling type-II predator-prey system," Journal of Mathematical Analysis and Applications, vol. 369, no. 2, pp. 555-563, 2010.

[20] V. Krrivan and J. Eisner, "The effect of the Holling type II functional response on apparent competition," Theoretical Population Biology, vol. 70, no. 4, pp. 421-430, 2006.

[21] C. Zhang, N.-J. Huang, and C.-X. Deng, "Dynamic behavior of positive solutions for a leslie predator-prey system with mutual interference and feedback controls," The Scientific World Journal, vol. 2014, Article ID 637026, 8 pages, 2014.

[22] Y. L. Cai, C. D. Zhao, and W. M. Wang, "Spatiotemporal complexity of a Leslie-Gower predator-prey model with the weak Allee effect," Journal of Applied Mathematics, vol. 2013, Article ID 535746, 16 pages, 2013.

[23] C. J. Xu and P. L. Li, "On the periodicity and global stability for a discrete delayed predator-prey model," International Journal of Mathematics, vol. 24, no. 10, Article ID 1350086, 2013.

[24] M. X. Liao, X. H. Tang, and C. J. Xu, "Stability and instability analysis for a ratio-dependent predator-prey system with diffusion effect," Nonlinear Analysis: Real World Applications, vol. 12, no. 3, pp. 1616-1626, 2011.

[25] C. Xu and Y. Wu, "Dynamics in a Lotka-Volterra predator-prey model with time-varying delays," Abstract and Applied Analysis, vol. 2013, Article ID 956703, 9 pages, 2013.

[26] G. Dunkel, "Single-species model for population growth depending on past history," in Seminar on Differential Equations and Dynamical Systems, vol. 60 of Lecture Notes in Mathematics, pp. 92-99, Springer, Heidelberg, Germany, 1968.

[27] D. Ye, M. Fan, and W. Zhang, "Periodic solutions of density dependent predator-prey systems with Holling type 2 functional response and infinite delays," Journal of Applied Mathematics and Mechanics, vol. 85, no. 3, pp. 213-221, 2005.

[28] Y. G. Sun and S. H. Saker, "Positive periodic solutions of discrete three-level food-chain model of Holling type II," Applied Mathematics and Computation, vol. 180, no. 1, pp. 353-365, 2006.

[29] R. Xu, L. Chen, and F. Hao, "Periodic solutions of a discrete time Lotka-Volterra type food-chain model with delays," Applied Mathematics and Computation, vol. 171, no. 1, pp. 91-103, 2005.

[30] Y. Li, "Positive periodic solutions of a discrete mutualism model with time delays," International Journal of Mathematics and Mathematical Sciences, no. 4, pp. 499-506, 2005.

[31] L. Nie, Z. Teng, L. Hu, and J. Peng, "Existence and stability of periodic solution of a predator-prey model with state-dependent impulsive effects," Mathematics and Computers in Simulation, vol. 79, no. 7, pp. 2122-2134, 2009.
[32] L.-L. Wang and W.-T. Li, "Periodic solutions and permanence for a delayed nonautonomous ratio-dependent predator-prey model with Holling type functional response," Journal of Computational and Applied Mathematics, vol. 162, no. 2, pp. 341-357, 2004.

[33] J. Wiener, "Differential equations with piecewise constant delays," in Trends in Theory and Practice of Nonlinear Differential Equations, vol. 90 of Lecture Notes in Pure and Applied Mathematics, pp. 547-552, Dekker, New York, NY, USA, 1984.

[34] W. Zhang, D. Zhu, and P. Bi, "Multiple positive periodic solutions of a delayed discrete predator-prey system with type IV functional responses," Applied Mathematics Letters, vol. 20, no. 10, pp. 1031-1038, 2007.

[35] M. Fazly and M. Hesaaraki, "Periodic solutions for predatorprey systems with Beddington-DeAngelis functional response on time scales," Nonlinear Analysis: Real World Applications, vol. 9, no. 3, pp. 1224-1235, 2008.

[36] M. Fan and K. Wang, "Periodic solutions of a discrete time nonautonomous ratio-dependent predator-prey system," Mathematical and Computer Modelling, vol. 35, no. 9-10, pp. 951-961, 2002.

[37] R. E. Gaines and J. L. Mawhin, Coincidence Degree and Nonlinear Differential Equations, Springer, Berlin, Germany, 1977.

[38] H. I. Freedman, Deterministic Mathematical Models in Population Ecology, Monographs and Textbooks in Pure and Applied Mathematics, Marcel Dekker, New York, NY, USA, 1980. 


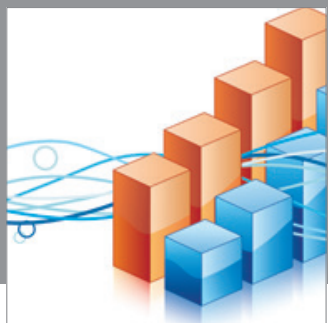

Advances in

Operations Research

mansans

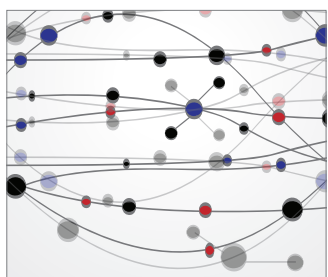

The Scientific World Journal
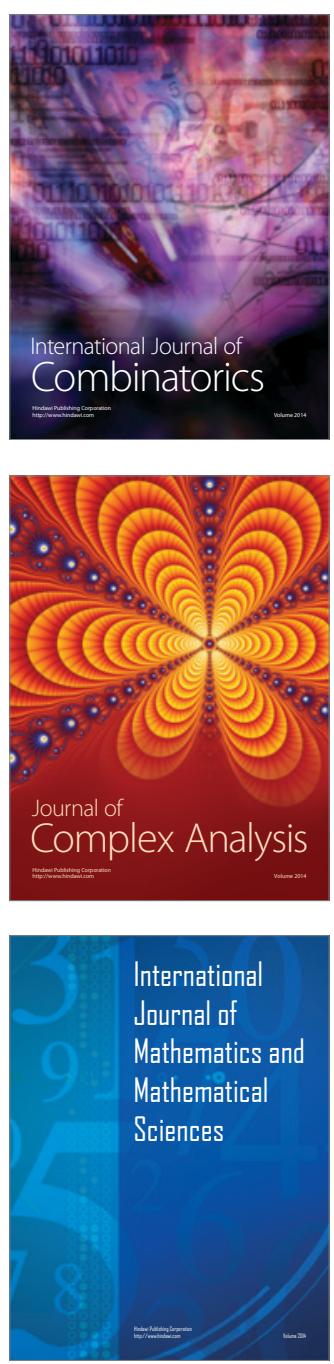
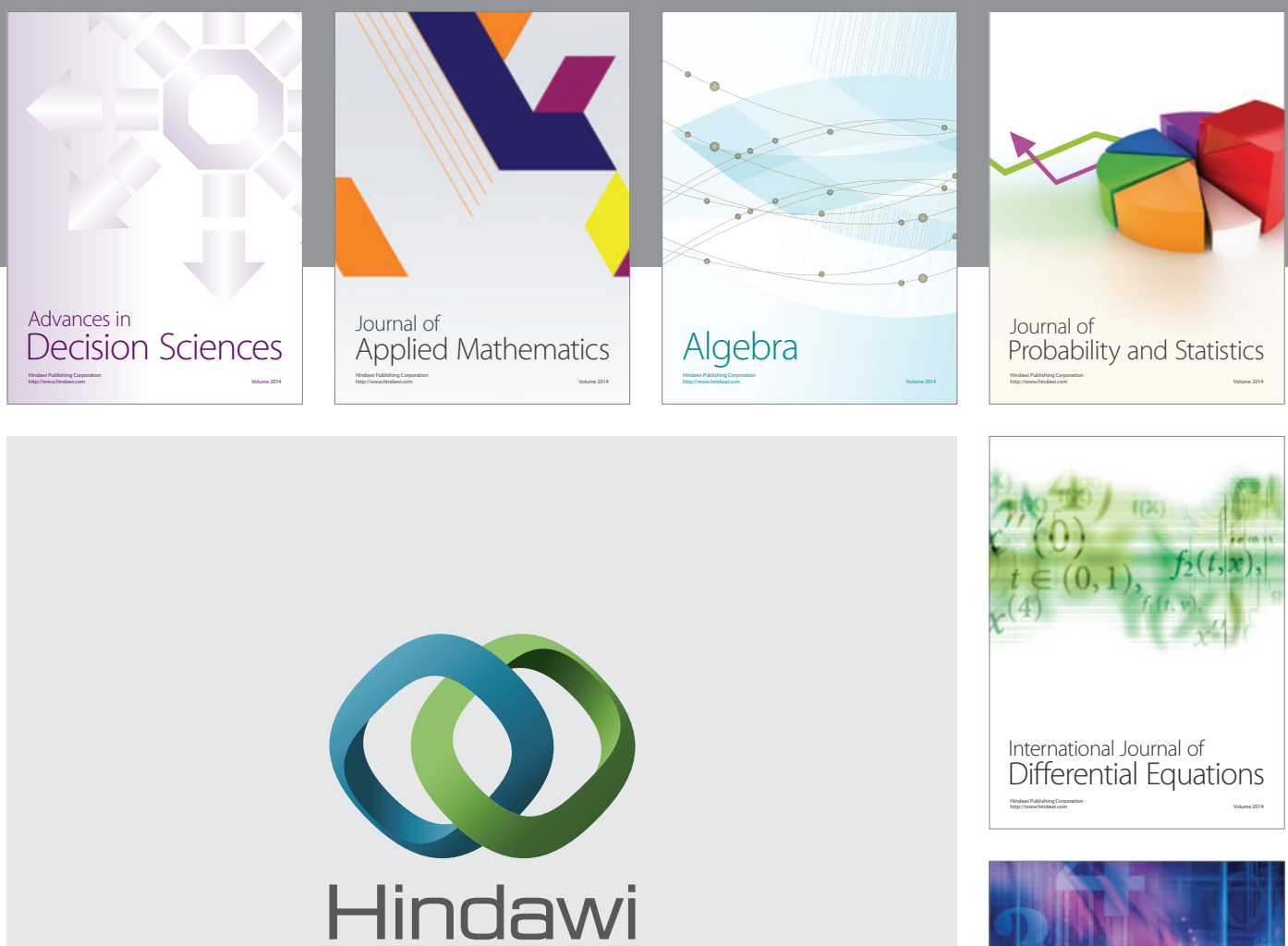

Submit your manuscripts at http://www.hindawi.com
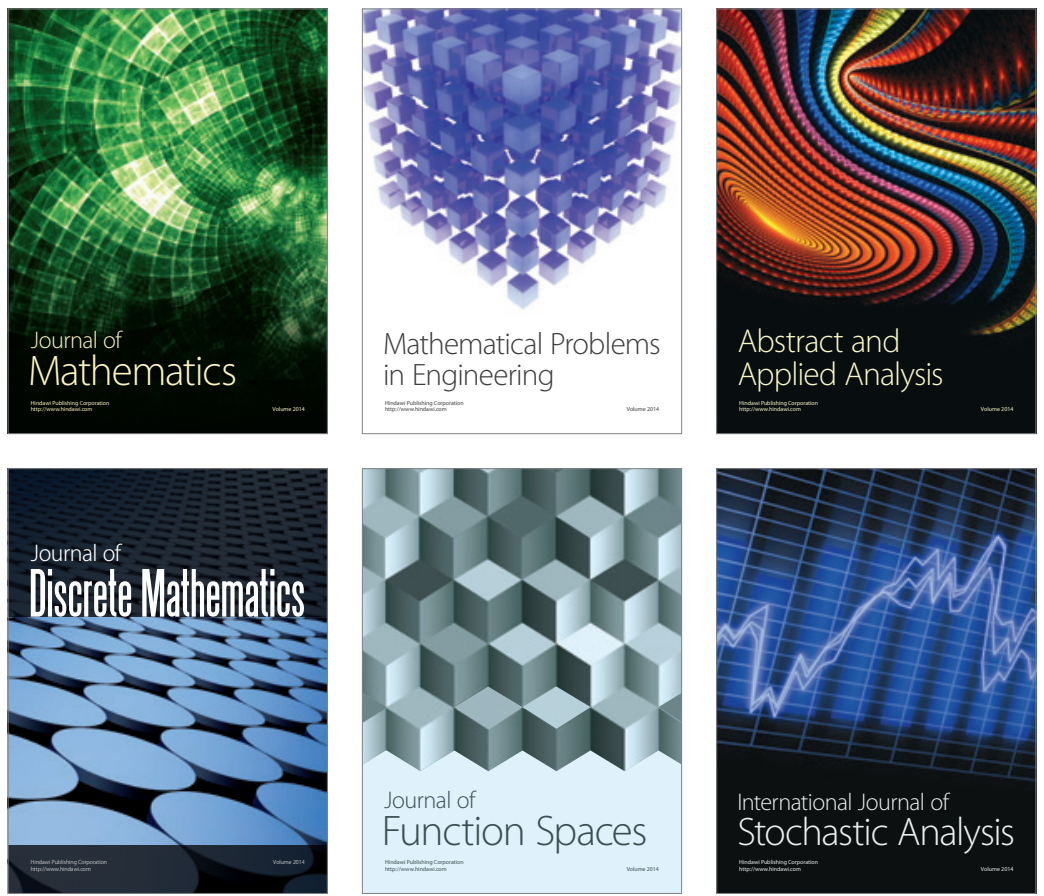

Journal of

Function Spaces

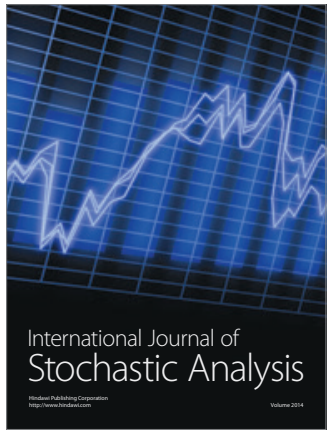

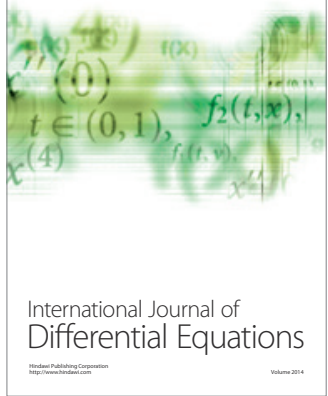
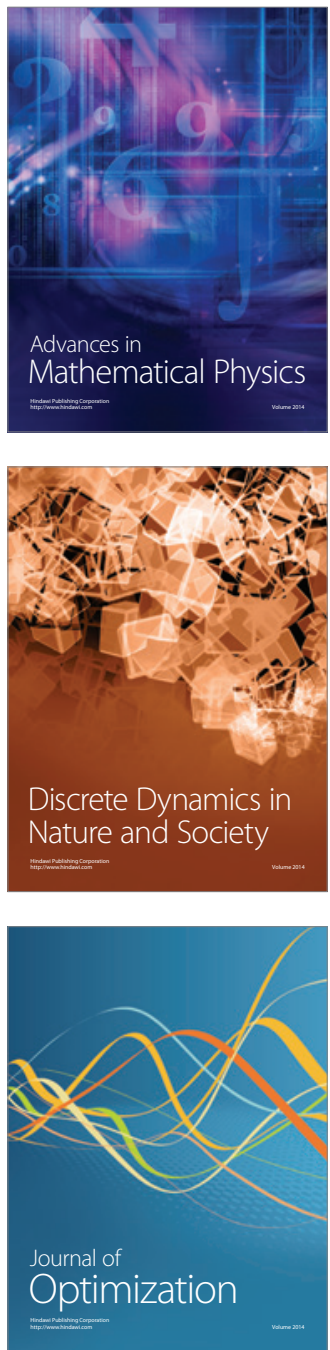